\title{
Longitudinal space charge assisted echo seeding of a free-electron laser with laser-spoiler noise suppression
}

\author{
Kirsten Hacker" \\ Zentrum für Synchrotronstrahlung, Technische Universität Dortmund, \\ Maria-Goeppert-Mayer-Straße 244227 Dortmund, Germany
}

(Received 19 May 2014; published 2 September 2014)

\begin{abstract}
Seed lasers are employed to improve the temporal coherence of free-electron laser (FEL) light. However, when these seed pulses are short relative to the particle bunch, the noisy, temporally incoherent radiation from the unseeded electrons can overwhelm the coherent, seeded radiation. In this paper, a technique to seed a particle bunch with an external laser is presented in which a new mechanism to improve the contrast between coherent and incoherent free electron laser radiation is employed together with a novel, simplified echo-seeding method. The concept relies on a combination of longitudinal space charge wakes and an echo-seeding technique to make a short, coherent pulse of FEL light together with noise background suppression. Several different simulation codes are used to illustrate the concept with conditions at the soft $\mathrm{x}$-ray free-electron laser in Hamburg, FLASH.
\end{abstract}

DOI: 10.1103/PhysRevSTAB.17.090702

PACS numbers: 07.85.Fv, 07.05.Fb

Short-wavelength, high-brightness light sources, like free-electron lasers (FELs) driven by particle accelerators, are in demand for experiments studying ultrafast processes in matter. The FEL community has pursued methods to improve the temporal coherence properties of the light [1-7] and to generate shorter, tunable FEL pulses [5-12]. When the temporal coherence of FEL light is determined by the shot noise of an electron beam, as in self-amplified spontaneous emission (SASE), it is poor [13-15], but if it is determined by an external seed laser, the FEL light takes on the excellent temporal coherence properties of the external laser in the region that has been seeded. However, if the seed pulse is short while the electron bunch is long, the noisy SASE background signal can overwhelm the seeded radiation, wiping out the benefits of the seed.

To reduce this noisy background, the FEL radiator is typically made short enough that the unseeded portion of the bunch does not reach saturation while the seeded portion does, but this can be limited in the case of short seeds with low seed power [1,5-7]. Alternatively, the electron bunch could be made short relative to the seed, but this puts challenging constraints on the synchronization between the femtosecond-scale seed and the electron bunch. Ideally, the electron bunch would be much longer than the femtosecond duration seed, so that with the best observed $25 \mathrm{fs}$ (rms) synchronization between electron beam and external laser in an FEL facility [16], the seed laser would hit the electron bunch on every shot, but then a

\footnotetext{
*kirsten.hacker@cfel.de

Published by the American Physical Society under the terms of the Creative Commons Attribution 3.0 License. Further distribution of this work must maintain attribution to the author(s) and the published article's title, journal citation, and DOI.
}

method is needed to suppress the SASE background in conjunction with the seeding of the short pulses.

As a relativistic seeded and bunched beam propagates along a drift, the microbunches experience a longitudinal space charge (LSC) impedance that modulates the energy of the beam in proportion to the peak current of the microbunches according to

$$
\Delta \gamma=\frac{|Z(k)|}{Z_{0}} \frac{I_{0}}{I_{A}} \rho_{k},
$$

where $Z_{0}=377 \Omega$ is the impedance of free-space, $I_{A}=$ $17 \mathrm{kA}$ is the Alfen current, $\rho_{k}$ is a small current perturbation at some wave number $k$, and $\gamma$ is the Lorentz factor. Depending on the strength of the impedance and the length of the drift, the energy spread of a microbunch can be increased to the point that it will no longer saturate in a FEL radiator of a reasonable length, thereby suppressing the lasing. This has been observed at the soft x-ray freeelectron laser in Hamburg, FLASH, in the form of a seeded microbunching instability or seeded longitudinal space charge amplification (LSCA) [17].

The seeding method described in this paper combines seeded LSCA SASE suppression with a short, echo-seeded pulse of temporally coherent FEL radiation. The idea builds upon the field of beam slicing techniques [8-12] by combining concepts from LSCA $[12,18,19]$ with echo enabled harmonic generation (EEHG), also known as echo-seeding [20,21]. Standard EEHG uses a modulatorchicane-modulator-chicane configuration with two seed lasers to modulate and filament the electron beam in the first stage and to modulate and bunch the beam in the second stage. The seeding method introduced here constitutes a new form of EEHG in that a LSC wake in a drift space provides the second-stage energy modulation that, in 
standard EEHG, is provided by a second external seed laser and modulator. The filamentation in conventional EEHG is also typically much stronger than what is used in this scheme. Particle tracking simulations were done to show that $0.5 \mathrm{GW}$ peak power, 10-50 fs (FWHM) duration pulses of SASE background-free, temporally coherent $8 \mathrm{~nm}$ radiation could be generated in the 27-m-long fixed-gap SASE undulator at FLASH.

In a possible implementation of the scheme at FLASH, an $800 \mathrm{~nm}$ Ti:sapphire laser pulse is sent through a BBO crystal frequency tripler in order to produce a $10-30 \mathrm{fs}$ (FWHM) $266 \mathrm{~nm}$ seed with up to several gigawatts of peak power. This is superimposed upon a picosecond long, $0.1 \mathrm{GW}$ pulse of either $800 \mathrm{~nm}$ or $266 \mathrm{~nm}$, depending on the desired seed laser harmonic. These seeds overlap with the electron bunch in the modulator, a 1.5-m-long undulator with six periods. The modulator is followed by a small magnetic chicane with up to $800 \mu \mathrm{m}$ of longitudinal dispersion at $900 \mathrm{MeV}$. The chicane is followed by a 27-m-long drift with an average electron beam radius of $150 \mu \mathrm{m}$ (rms). After the drift comes a second, smaller chicane followed by the 27-m fixed gap radiator undulator with a period of $27.3 \mathrm{~mm}$. The electron beam energy is $900 \mathrm{MeV}$ and the initial uncorrelated energy spread is $150 \mathrm{keV}$ (rms). The layout of the scheme prior to the FEL radiator is shown in Fig. 1.

By constructively superimposing a picosecond duration, low-intensity background seed pulse upon a 10-30 fs (FWHM) duration, high-intensity spike, the energy of the electron bunch will be sinusoidally modulated as shown in Fig. 2(a). When an energy modulated particle bunch travels through a dispersive section, like a magnetic chicane, the high-energy particles travel a shorter path than the low-energy particles, producing a longitudinally microbunched structure with a periodicity equal to the seed wavelength. A chicane that will maximally bunch the electrons with a small energy modulation will overfold the electrons with a larger energy modulation. Since the tails have a smaller amplitude energy modulation compared to the electrons in the spike, the tails can be highly bunched with a high peak current, while the spike will be folded over with a lower peak current [Fig. 2(b)].

In Fig. 3, short slices of the electron beam from Fig. 2 are depicted qualitatively at key points in the seeding process.

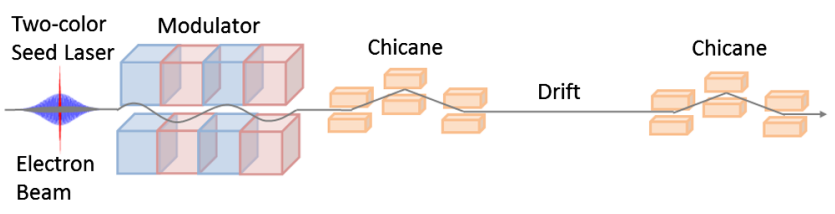

FIG. 1. Layout of seeding scheme prior to the FEL radiator. A modulator is followed by a bunch compressor chicane and a 27-m-long drift section with tightly focused electron beam optics. After the drift, there is an additional chicane directly prior to the FEL radiator.
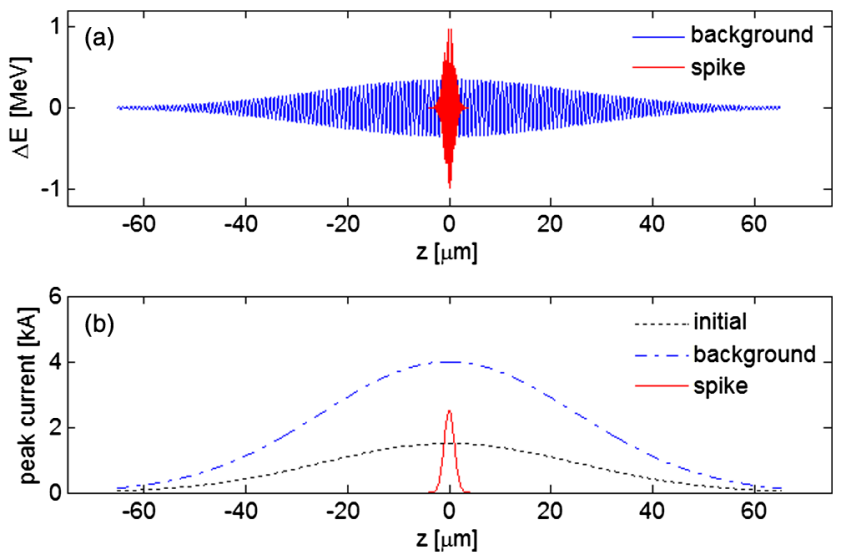

FIG. 2. Longitudinal energy (a) and microbunch peak current (b) distribution prior to the drift. The central spike is responsible for LSC-EEHG seeding, while the longer background pulse is responsible for suppression of the lasing from SASE. The central spike of energy modulation is initially folded over while the less energy-modulated portion is maximally bunched in the first chicane. This leads to different amounts of peak current in different microbunches.

Figures 3(a) and 3(e) depict the initial energy modulations of the spike and the background and Figs. 3(b) and 3(f) depict the density modulation after the first chicane. In the subsequent drift, the high-peak current microbunches in the tails will experience stronger LSC wakes [Fig. 3(g)] than the lower peak current microbunches in the folded over and filamented spike [Fig. 3(c)]. After the drift, while the filamented and energy modulated electrons in the spike can be bunched by the chicane prior to the radiator, making an echo-seeded beam distribution with high harmonic content [Fig. 3(d)], the electrons in the tails would get folded over and debunched in the same chicane, smearing out the charge and suppressing the harmonic content [Fig. 3(h)]. In the subsequent radiator undulator, the femtosecond echoseeded spike will radiate coherently at a harmonic of the seed laser, while the lasing will be suppressed in the picosecond tails. This progression from seeded energy modulation to LSC energy modulation and final bunching/debunching will be referred to as LSC-EEHG seeding in the spike and lasing suppression in the tails.

Alternatively, by shifting the phase of the spike relative to the background pulse so that the interference is destructive instead of constructive, when the amplitude of the spike is equal to that of the background, a seed with a hole in the middle is produced. The lasing would be suppressed everywhere except for in the hole. A direct comparison of the coherence properties of such a masked SASE pulse with an LSC-EEHG seeded pulse could be made by switching the properties of the spike between two states.

While Fig. 3 shows the simpler case of a single-color seed, a two-color seed allows the method to seed higher harmonics. By using a background wavelength which is longer than the spike wavelength, the LSC-EEHG seeded 


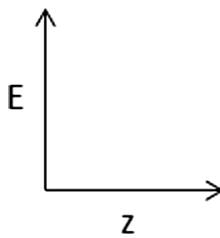

(a)

(b)

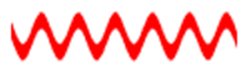

seed modulation

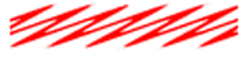

folding (c)

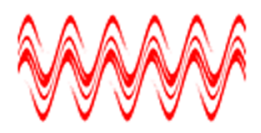

LSC wake modulation (d)

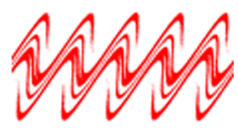

bunching (e)

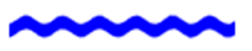

seed modulation (f)

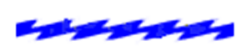

bunching (g)

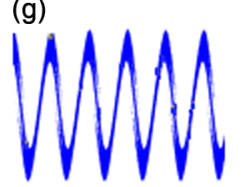

LSC wake modulation (h)

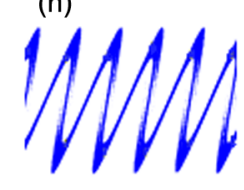

FIG. 3. Longitudinal phase space of LSC-EEHG seeded (a)-(d) and lasing suppressed (e)-(h) electron beam slices at different stages of the seeding process. Although two-color seeds are used in subsequent simulations, the simpler case of a single seed modulation wavelength was used here to illustrate the mechanism.

spike can be folded over more dramatically while the background particles are bunched. For two-color operation, it is possible to use either two sequential undulators tuned to be resonant at the different wavelengths, or a single modulator undulator tuned to be resonant at a subharmonic of the shorter wavelength seed. This requires more peak power for the shorter wavelength seed compared to a modulator that is not tuned to a subharmonic; however, for short, intense seed pulses, this is technically feasible.

These simulations were produced with three different particle tracking codes, starting with a quick 1D code, based on [22], with the LSC impedance given by [23]

$$
Z_{\mathrm{LSC}}(k)=\frac{i Z_{0}}{\pi k r_{b}^{2}}\left[1-\frac{k r_{b}}{\gamma} K\left(\frac{k r_{b}}{\gamma}\right)\right],
$$

where $r_{b}=0.85\left(\sigma_{x}+\sigma_{y}\right)$ is the radius of a uniform, round beam, $K$ is a modified Bessel function of the second kind, $\gamma$ is the Lorentz factor, and $k r_{b}<\gamma / 2$. Particle motion is produced at each step according to $L / \gamma^{2}$, where $L$ is a $0.5 \mathrm{~m}$ step. The initial energy modulation produced by the seed is calculated for a given seed waist, peak power, and number of undulator periods. Optimal operation points were observed in a range of $10 \%$ around $2 \lambda_{\text {seed }}=\eta \Delta E / E$ where $\eta$ is the longitudinal dispersion of the chicane and $\Delta E$ is the peak-to-peak energy modulation. For $2 \times 10^{6}$ particles, the 1D code executes within seconds on a desktop PC. It was cross-checked with a 1D and a 3D periodic LSC code [24] with 16000 particles/nm and a run time of $10 \mathrm{~min}$. The 3D periodic LSC code, with a period defined by the seed wavelength, was benchmarked with the space charge particle tracker ASTRA 3D, which relies on a RungeKutta integration, is not periodic, and takes a day to run [25]. The quick 1D code with 1000 particles/nm was used to search for viable operation regions, while the periodic 3D code was used to make more realistic predictions about the harmonic content of the beam.

A 3D quantitative picture of the longitudinal phase space resulting from a two-color scheme is shown in Fig. 4 with longitudinal slices from the lasing suppressed (left) and seeded (right) portions of the beam. The beam energy was $900 \mathrm{MeV}$ with an $800 \mathrm{~nm}$ seed in the tails and a short, more intense $266 \mathrm{~nm}$ seed. The microstructures in 3D are partially longitudinally smeared out compared to a $1 \mathrm{D}$ simulation.

Taking the Fourier transform of a single cycle of the peak current distribution shown in Fig. 4 gives the bunching factor, which can be written in terms of total number of particles $N_{0}$, wave number $k$, and 1D particle density $\mathrm{N}(\mathrm{z})$ as

$$
b(k)=\frac{1}{N_{0}} \int_{0}^{L} e^{i k z} N(z) \mathrm{d} z .
$$

The bunching factor is plotted as a function of harmonic number in Fig. 5. At high harmonics of the $266 \mathrm{~nm}$ seed wavelength, the LSC-EEHG seeded portion of the bunch has harmonic content at the 33rd harmonic of $266 \mathrm{~nm}(8 \mathrm{~nm})$, while the lasing suppressed portion does not.

The spectral peaks in Fig. 5 can be shifted to lower and higher harmonics when the longitudinal dispersion, $R_{56}$, of a chicane is adjusted by $5-10 \mu \mathrm{m}$ in order to accommodate seeding at different wavelengths. The amplitude of the LSC energy modulation can be tuned by changing either the transverse beam size in the drift, per Eq. (2) or by tuning the transfer matrix elements $R_{53}=\Delta y^{\prime} / \Delta z$ and $R_{54}=\Delta y / \Delta z$ in the drift. These terms can be expressed in quantities that are easy to measure through beam-based dispersion measurement tools: $R_{53}=-R_{46}=\Delta y^{\prime} / \Delta E$ and in the middle of a bump $R_{54}=-R_{36}=\Delta y / \Delta E$.

The bunching factor of 0.005 at the 33rd harmonic appears concerning, since it is much smaller than the bunching factors that are used for conventional seeding schemes with shorter, $10 \mathrm{~m}$ undulators, but it is still well above the 0.0001 start-up noise for SASE [26], and since the undulator is $27 \mathrm{~m}$ long, it is still effective. For comparison, if an identical electron bunch with a $1 \mathrm{MeV}$ 

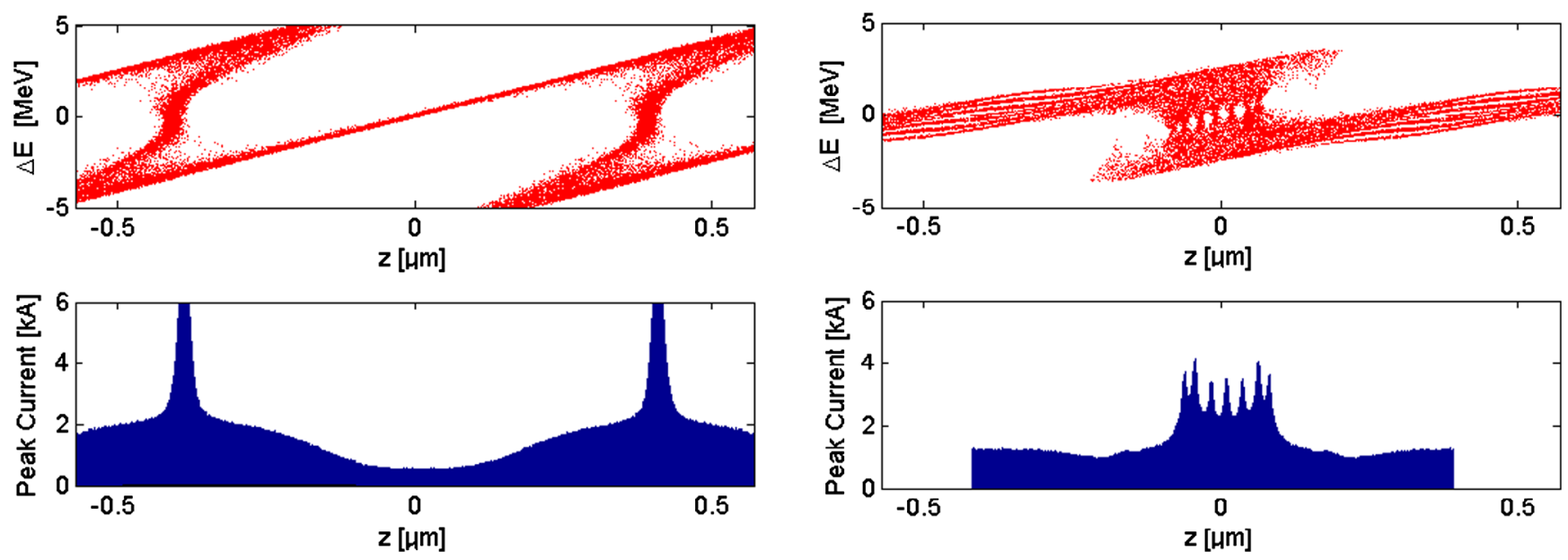

FIG. 4. 3D longitudinal phase space after last chicane for LSC-EEHG seeded (right) and lasing suppressed (left) portions of the beam. Seeding was done with $800 \mathrm{~nm}$ in the tails (left) and with a combination of $800 \mathrm{~nm}$ and $266 \mathrm{~nm}$ in the central spike (right). The average beam radius in the $26 \mathrm{~m}$ drift section was $150 \mu \mathrm{m}(\mathrm{rms})$, the longitidutinal dispersion of the first and second chicanes was $485 \mu \mathrm{m}$ and $85 \mu \mathrm{m}$, respectively, the initial peak current was $1.5 \mathrm{kA}$, and the beam energy was $900 \mathrm{MeV}$ with a $150 \mathrm{keV}$ (rms) initial slice energy spread. The initial energy modulation was $350 \mathrm{keV}$ in the $800 \mathrm{~nm}$ background and $1 \mathrm{MeV}$ in the $266 \mathrm{~nm}$ seeded region. The rms energy spread of the seeded portion is $1.5 \mathrm{MeV}$ and the rms energy spread of the lasing suppressed portion is $4 \mathrm{MeV}$.

(pp) energy modulation were used to do HGHG seeding [1] with a 10-m-long radiator, the bunching factor would become unusable above the 10th harmonic.

The harmonic content from Fig. 5 and the rms energy spreads and beam conditions from Fig. 4 can be used to predict the length of radiator undulator required for saturation of the FEL to occur for the seeded and lasing suppressed slices of the electron bunch. This prediction is made with the Xie 3D model [27] for FEL radiation combined with a formula for the sensitivity of the saturation length to an initial bunching factor, Eq. (4) of [26],

$$
L_{\mathrm{sat}}=L_{g} \cdot \log \left(1 / \alpha \cdot b_{n}\right)
$$

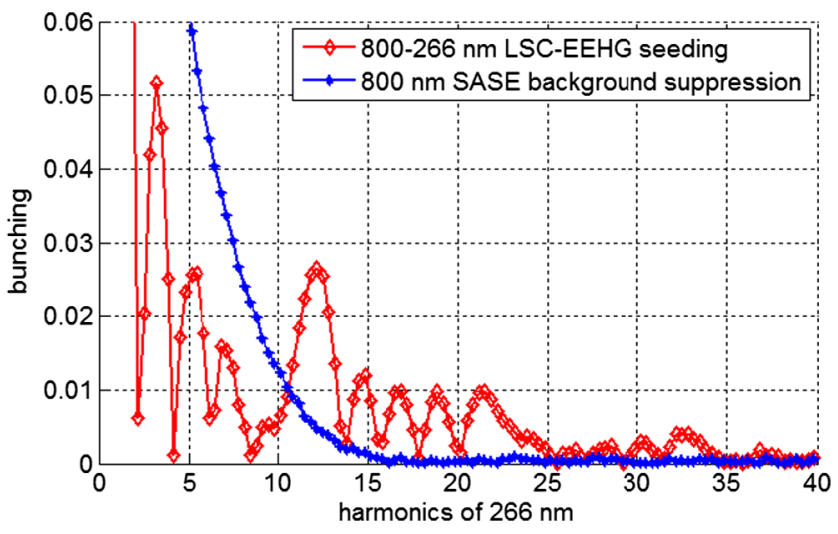

FIG. 5. 3D beam harmonics of $266 \mathrm{~nm}$ after last chicane for seeded and lasing suppressed portions of the beam from Fig. 4. Seeding was done with $800 \mathrm{~nm}$ in the tails and with a combination of $800 \mathrm{~nm}$ and $266 \mathrm{~nm}$ in the central spike. $8 \mathrm{~nm}$ is the 33rd harmonic of $266 \mathrm{~nm}$. where $L_{g}$ is the gain length without seeding, $\alpha$ is the $1 / 9$ fraction of noise power coupled into the dominant mode, and $b_{n}$ is the bunching factor at the $n$th harmonic of the seed. This method was chosen instead of the FEL code Genesis [28], due to the FEL bucket length in Genesis being of the same length scale as the seeded microbunches.

At FLASH, the $900 \mathrm{MeV}$ beam energy corresponds to $8 \mathrm{~nm}$ radiation from the 27-m-long fixed gap undulator (33rd harmonic of $266 \mathrm{~nm}$ ). For an emittance of $1.5 \mathrm{~mm}$ mrad, a peak current of $1.5 \mathrm{kA}$, and an energy spread of $0.15 \mathrm{MeV}$ (rms), the SASE saturation length is $16.6 \mathrm{~m}$ and the saturation power is $2.6 \mathrm{GW}$. For the lasing suppressed conditions from Fig. 4, with an rms energy spread of $4 \mathrm{MeV}$, the saturation length would be $122 \mathrm{~m}$. For the seeded conditions from Fig. 4, with a bunching factor of 0.005 and an rms energy spread of $1.5 \mathrm{MeV}$, saturation would be achieved within the $27 \mathrm{~m}$ length of the undulator with a peak power of $0.5 \mathrm{GW}$. Without the SASE background suppression, the seeded FEL pulse energy would be smaller than the SASE pulse energy and the seeded FEL pulse would be longer than the $10 \mathrm{fs}$ (FWHM) seed, due to slippage of the FEL radiation through the bunch.

Seed laser amplitude fluctuations typically produce $1 \%$ (rms) energy modulation variation and this causes 5\%-10\% jitter of the $8 \mathrm{~nm}$ LSC-EEHG bunching factor and 20\%$30 \%$ jitter for conventional EEHG at $8 \mathrm{~nm}$. However, in contrast to conventional EEHG, LSC-EEHG is affected by small changes in the peak current of the macrobunch. With $5 \%$ (rms) peak current jitter, the bunching factor at $8 \mathrm{~nm}$ changes by $5 \%$. With $25 \%$ jitter, the bunching factor varies by $100 \%$. Taken altogether, the LSC-EEHG bunching factor jitter is potentially better than that of standard EEHG.

In conclusion, LSC-EEHG is a new seeding technique that can be combined with a method to suppress the SASE 
background lasing, so that $10 \mathrm{fs}$ (FWHM) pulses of seeded FEL radiation can be generated with no SASE background and relaxed synchronization requirements between seed and electron bunch. Without the SASE lasing suppression, LSC-EEHG offers advantages over traditional EEHG in that a second modulator and seed laser are not required for the second stage of EEHG, removing the inherent synchronization and overlap difficulties at the cost of a more space consuming design. While not ideal for a compact FEL, this concept can enhance the performance of existing infrastructure, using, for example, laser heater infrastructure to seed a first EEHG stage and relying on LSC to provide the second stage energy modulation after compression, or, as in the case of FLASH, using the seeding infrastructure upstream of the fixed gap SASE undulator.

The author would like to thank M. Dohlus for access to his 3D periodic LSC simulation code and Professors S. Khan and J. Rossbach for reading and commenting on the paper. This work was supported by BMBF Grant No. 05K13PE3 and DESY.

[1] E. Allaria et al., Nat. Photonics 7, 913 (2013).

[2] Z. Zhao et al., Nat. Photonics 6, 360 (2012).

[3] D. Xiang et al., Phys. Rev. Lett. 108, 024802 (2012).

[4] J. Amann et al., Nat. Photonics 6, 693 (2012).

[5] T. Ishikawa et al., Nat. Photonics 6, 540 (2012).

[6] L. Giannessi et al., Phys. Rev. Lett. 110, 044801 (2013).

[7] S. Ackermann et al., Phys. Rev. Lett. 111, 114801 (2013).

[8] E. L. Saldin, E. Schneidmiller, and M. Yurkov, Nucl. Instrum. Methods Phys. Res., Sect. A 507, 439 (2003).

[9] A. A. Zholents and W. M. Fawley, Phys. Rev. Lett. 92, 224801 (2004).

[10] E. L. Saldin, E. A. Schneidmiller, and M. V. Yurkov, Phys. Rev. ST Accel. Beams 9, 050702 (2006).
[11] Y. Ding, Z. Huang, D. Ratner, P. Bucksbaum, and H. Merdji, Phys. Rev. ST Accel. Beams 12, 060703 (2009).

[12] M. Dohlus, E. A. Schneidmiller, and M. V. Yurkov, Phys. Rev. ST Accel. Beams 14, 090702 (2011).

[13] R. Bonifacio, L. De Salvo, P. Pierini, N. Piovella, and C. Pellegrini, Phys. Rev. Lett. 73, 70 (1994).

[14] P. Pierini and W. M. Fawley, Nucl. Instrum. Methods Phys. Res., Sect. A 375, 332 (1996).

[15] E. L. Saldin, E. A. Schneidmiller, and M. V. Yurkov, Opt. Commun. 148, 383 (1998).

[16] I. Grguras et al., Nat. Photonics 6, 852 (2012).

[17] C. Lechner et al. (to be published).

[18] E. A. Schneidmiller and M. V. Yurkov, Phys. Rev. ST Accel. Beams 13, 110701 (2010).

[19] M. Dohlus, E. A. Schneidmiller, and M. V. Yurkov, Phys. Rev. ST Accel. Beams 14, 090702 (2011).

[20] G. Stupakov, Phys. Rev. Lett. 102, 074801 (2009).

[21] D. Xiang and G. Stupakov, Phys. Rev. ST Accel. Beams 12, 030702 (2009).

[22] R. A. Bosch, K. J. Kleman, and J. Wu, Phys. Rev. ST Accel. Beams 11, 090702 (2008).

[23] Z. Huang, M. Borland, P. Emma, J. Wu, C. Limborg, G. Stupakov, and J. Welch, Phys. Rev. ST Accel. Beams 7, 074401 (2004).

[24] M. Dohlus, E. Schneidmiller, M. V. Yurkov, C. Henning, F. J. Grüner, E. Esarey, C. B. Schroeder, W. P. Leemans, K. W. D. Ledingham, and D. A. Jaroszynski, Proc. SPIE 8779 87791T, (2013).

[25] K. Flottmann, S. M. Lidia, and P. Piot, in Proceedings of the 20th Particle Accelerator Conference, Portland, OR, 2003 (IEEE, New York, 2003), p. 3500.

[26] G. Dattoli, A. Doria, L. Giannessi, and P. L. Ottaviani, Nucl. Instrum. Methods Phys. Res., Sect. A 507, 388 (2003).

[27] M. Xie, in Proceedings of the Particle Accelerator Conference, Dallas, TX, 1995 (IEEE, New York, 1995), p. 183.

[28] S. Reiche, Nucl. Instrum. Methods Phys. Res., Sect. A 429, 243 (1999). 